\title{
Design of Living Beings Electrotherapy Instrument System
}

\author{
Chunhua Zhao ${ }^{a}$, Xiaoqiang $\mathrm{Ji}^{\mathrm{b}}$, Chunyu Ning ${ }^{\mathrm{c}}$, Danfei Huang ${ }^{\mathrm{d}}$ \\ School of Life Sciences and Technology, Changchun University of Science and Technology, \\ Changchun 130022, China \\ azch_gdy@163.com, bzuoanmulan@163.com, ${ }^{\mathrm{c}}$ yeningcy@163.com, ${ }^{\mathrm{d}}$ Huangdf@cust.edu.cn.
}

\begin{abstract}
Keywords: MCU; electrotherapy instrument; living beings.
\end{abstract}
\begin{abstract}
A low frequency living beings electrotherapy instrument is designed which is based on the transcutaneous electrical nerve stimulation. This system uses ATmega32 (MCU) to control its output and display. The hardware part includes the keyboard, the LCD, the alarm circuit and output circuit .The other part is software, which includes keyboard module, LCD module, pulse creating module and so on. The human-computer interaction can be realized well by the keyboard and LCD module. The pulse creating module controls the frequency, the duty-ratio and time of treatment.
\end{abstract}

\section{Introduction}

In the area of treatment, physical therapy is a common method of treatment. It is wide application because of the course of treatment without pain, little side effects and significant effect on certain diseases. Investigation shows that the fixed frequency signal is long applied to the stimulation of the human body, the signal will weaken the effect of the human body treatment ${ }^{[1]}$. Therefore, it is significant for the stimulation of the body that the output signal frequency is independent, which can be well adapted to overcome the ability of human tissue, make the patient obtain the optimal therapeutic effect. The output signal of traditional physical therapy instrument has a certain regularity, which can make the body produce the adaptability and reduce the effect.

The core of living beings electrotherapy instrument is the output signal waveform generation module, which relies on Atmega32 to achieve. The PWM module of Atmega32 can control the shape, frequency and amplitude of the output signal, and the output waveform can be generated randomly, applicable symptoms. The system can well solve the shortage of traditional

electrotherapy instrument system, such as the treatment of adaptability of output signal, polarization voltage smaller, patient comfort and simple operation etc.. Therefore, it has a certain practical value to research and development of living beings electrotherapy.

\section{Electrotherapy principle}

The human body is a good conductor of electricity, has certain conductivity. During the treatment, the pulse current is applied to the deep body tissues and stimulate tissue to produce a variety of regular and irregular shrinkage ${ }^{[2]}$. Such action by the organization can reduce the pain sensation, promote parts of the organization metabolism, but also has anti-inflammatory, removing blood stasis, adjusting the endocrine and metabolic disorders, improving local tissue blood circulation, eliminating excess fat, dredging the channel and other effects $^{[3]}$. The pulse signal frequency is lower than $1000 \mathrm{~Hz}$ is defined as the low frequency signal in medicine. The use of low frequency electrical stimulation of tissue to recuperate the body way is referred to as low frequency electrotherapy. Low frequency electrical signals applied to human tissue, stimulates the human tissue, which can accelerate the blood flow of partial tissue and alleviate the pain ${ }^{[4]}$. Treatment principle of the system is based on transcutaneous electrical nerve stimulation therapy ${ }^{[5]}$. The low frequency electrical signal is inputted to the human body by the skin and plays a role in healing and alleviate pain, which is known as TENS. TENS is widely used clinically, which can promote the local blood circulation, alleviate the pain and promote the development of bones. 
People know about more the pain mechanism along with the progress of electronic and medical knowledge and the improving of people living conditions. Firstly the meridian theory thinks that the disease occurrence and development are related the acupoints of main and collateral channels. Later, the research indicates that the main and collateral channels and acupoints have the characteristic of potential high and low impedance and the impedance can change when the electrical signal effects them, which can make the human body appear acid, hemp, pain, swelling and other physiological phenomena. Therefore, the meridian and points which can reflect the function of muscle tissue, organ or physical condition should be chosen, the potential of the body can quickly restore balance, the meridian smooth, and the body is healthy by imposing its corresponding pulse signal.

\section{The overall design of the system}

The structure diagram of the system is shown in figure 1. It mainly consists of two parts: based on the ATmega32 control circuit and PWM interface circuit. The core of the control circuit is ATmega32, which has abundant resources and general equipment interface and includes key module, LCD module, PWM output module, sampling module, alarm module. The PWM Interface circuit includes filter circuit and power amplifier circuit.

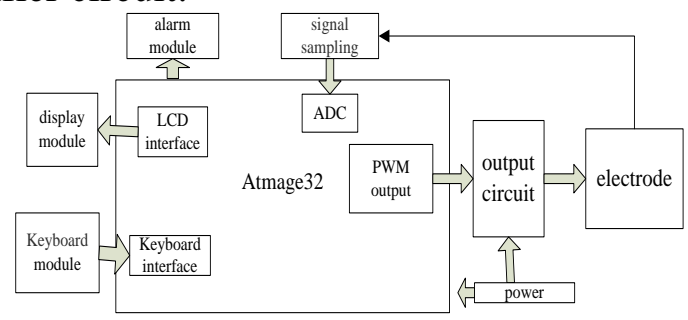

Fig1. structure diagram of the system

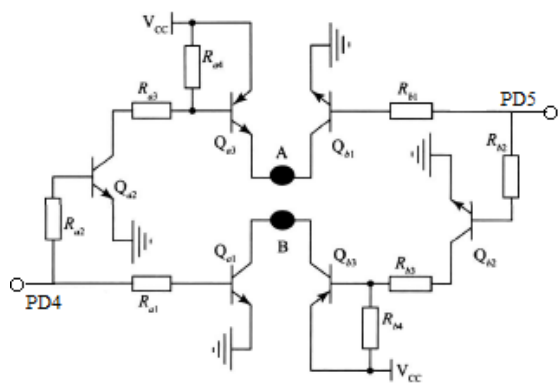

Fig2. output circuit

\section{Hardware design of the electrotherapy}

Control chip. ATmega32 microcontroller comes with 10 ADC conversion and 16-bit timer / counter, which can be defined as a single-ended or differential input mode when using the ADC, which can be defined as a single-ended or differential input mode, and the timer can realize functions of PWM and frequency divider. So the system can not the single A/D chip. The watchdog circuit integrated within Atmega32, which can effectively enhance the reliability of electrotherapy system.

Human interface. The keyboard consists of two parts: the function keys and the numerical keys which uses non coding keyboard. The two part keys are composed of mechanical contacts, which work together to control the system in good working condition. Function keys include: frequency, duty cycle, the timing time setting. The function keys select the PD2 pin, which is used for interrupt mode. The numerical keys use a matrix structure, which select query mode to realize the input values.

The display module select CM320240 Graphic LCD display, which is a size of $320 \times 240$ display screen. The control part of the screen is RA8835 which is used to display the data lines are 4, the fast data transfer can be realized through its. It has strong graphics capabilities, not only support the text information display, but also support the graphical display and a mix of both information display. The interface circuit with MCU is simple and includes full. The production technology of the 
control part is SMT process and its structure is stability and can use a long time. The PD0, PD1 of the MCU can control write / read operation, the PD3 can control select, and the PC0-PC7 are the data output ports.

Control circuit. It can realize the real time sampling by the without a sense resistor $\mathrm{R}$ series in the output circuit, the resistance of the resistance is very small, so that the accurate data of output current can be obtained. Then the sampled current signal will be sent to the ADC of the microcontroller for A /D conversion, and stored in the EEPROM of the microcontroller in order for the LCD display. At the same time the extracted data are compared with the set threshold value, the system can start the alarm circuit for the reminder when there is tolerance.

Output module. The PD4, PD5 port of the ATmega32 can output the PWM pulse signal which meets the requirements. The signal depends on the specific algorithms and pulse parameters set by the keyboard.The output signal of the microcontroller is processed by the pulse output circuit and then turned into ion by the electrode and treats directly on the painful area. Figure 2 is a circuit diagram of a pulse output module.PD4 and PD5 of the figure 2 output PWM pulse signal, which are controlled by the ATmega32. After conditioning and amplification the signal is output by A and B electrode. VCC is a low voltage DC power which is provided by the power supply module.

Alarm module. It can controlled by the Atmage32 to drive the buzzer to prompt treated shut down the machine, when the treatment time to or output circuit current exceeds the set value.This can provide security.

\section{Software design of the electrotherapy}

Software system can be divided into five modules: the main program, PWM wave generation module, keyboard module, LCD module and the signal sampling module. The flow chart of the system is shown in Figure 3.

PWM wave generation module. Electrotherapy pulse signal of the system is a square wave pulse signal which is produced by MCU internal T/C1 when working in the PWM output pattern. It includes a plurality of output waveforms of control subroutine, such as pulse frequency adjustment, the pulse intensity adjustment, treatment time selection.

Keyboard module. The keyboard module includes keyboard scanning and key handler.Keyboard scanning program will continue to run to realize real-time monitoring of the keyboard circuit so that the system can meet the needs of users. The control module executes the relevant operation according to the query to the key value and the corresponding handler.

LCD module. The LCD module can display the waveform and the control parameters of the output pulse signal. Therefore, the LCD module function consists of two parts: the waveform and text. Waveform display can achieve the collected output pulse signal real-time display. Text display can achieve the signal parameters display which are input by the user through the key module.

Signal sampling module. The current of the electrotherapy output signal are sampled real-time by the signal sampling module and converted to digital quantity by the ADC. The comparison of the current value and the set current value can control the output pulse signal of system and keep the system stable. At the same time, the sampled data are sent to the liquid crystal display module.

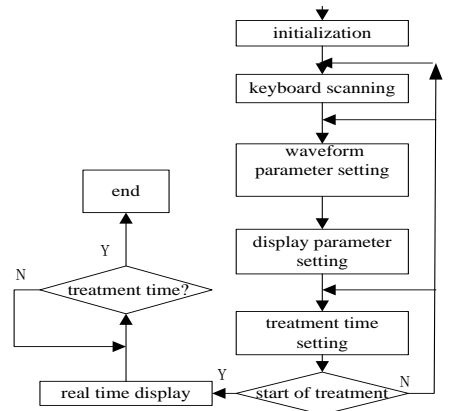

Fig3. flow chart of the system 


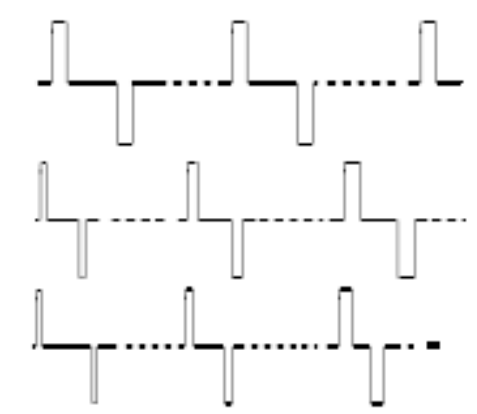

Fig4. electrical stimulation waveform

\section{Electrotherapy waveforms}

The most basic electrical pulse of the electrotherapy generate is a square wave pulse signal. When power has the stimulation, it makes the muscle contraction and stretch, and then improve the blood and lymph circulation parts, play anti-inflammatory and analgesic effect. System uses pulse width between $10 \mu \mathrm{s} \sim 0.3 \mathrm{~ms}$, during the width of the square-wave the current can extend nerves duration, reduce their pain, and result in analgesic effect.

The different frequency and different duty cycle of the pulse are combined by the system based on the low frequency electrical characteristics and avoiding the adaptability of human body. There are 36 kinds of pulse combination system, for different parts and different diseases using. It can recycle, also choose a single pulse. Figure 4 is used to electrical stimulation waveform.

\section{Summary}

Living beings electrotherapy is a commonly used instrument of family health care. It has the characteristics of low power consumption, small volume, high cost performance, simple operation, which are the trend of development in the future. It is based on transcutaneous electrical stimulation theory and uses the timer module of Atmage32 to realize the output pulse signals which are processed to effect on the human body by the electrode. It can stimulate relevant organizations and play a role in treatment. The electrotherapy system has good human-machine interface and easy operation.

\section{References}

[1]Limin Zhang. Design of multi frequency electrotherapy instrument based on FPGA.J. Inner Mongolia Science Technology \& Economy.2008(1):91-92.

[2]Ronald J. Weinstock. Method and Apparatus for Resistivity Measurement, Detection and Treatment in Living Tissue.P.US:2010/0324627A1,2010-12-23.

[3]Tiebin Yan,Shuguang Cheng. Low frequency pulse electrical stimulation paralysis therapeutic instrument based onwalking pattern.J. Chinese Medical Equipment Information,2010,16(2):1-4.

[4]Fuqiang Liu. Analysis and design of electric pulse treatment instrument circuit.J.Journal of Taiyuan Normal University.2008,7(3):93-95.

[5]Xilin Xu,Yueping Zhou,Xudong Guo etal.Development of a FES therapeutic apparatus based oncerebrovascular disease patient.J.Computer Measurement\&Control.2010,18(2):482-484. 